\title{
Description de la femelle d'Hartertia natalensis Monnig, 1931 et redescription des spicules du mâle
}

\author{
par J. A. CRUZ E SILVA \\ [Centre de Zoologie de la Junta de Investigações do Ultramar, \\ et Ecole Supérieure de Médecine Vétérinaire, Lisbonne] (1)

\section{Résumé} \\ Hartertia natalensis Mönnig, 1931, espèce trouvée au Natal \\ et connue seulement par les mâles, est retrouvée au Mozambique. \\ La femelle est décrite; la morphologie des spicules est précisée.
}

\section{Summary}

Hartertia natalensis Mönnig, 1931, a species found in Natal and known by its males only, has been collected in Mozambique. The female is described in this paper and full details are given on the morphology of spicules.

Trois lots d'Hartertia comprenant des mâles et des femelles ont été récoltés chez Lissotis melanogaster notophila Oberholser 1905, au Mozambique (2).

Un seul spécimen mâle était dépourvu d'ailes cervicales et correspondait à l'espèce Hartertia zuluensis Mönnig, 1931. Tous les autres mâles et femelles avaient des ailes cervicales ; ce caractère, ainsi que l'existence d'une tête saillante nettement séparée du reste du corps par un cou plus étroit, et les deirides, déportées sur la face dorsale, ont permis d'identifier ces spécimens à Hartertia natalensis Mönnig, 1931.

Cette espèce étant décrite par Mönnig uniquement à partir de deux mâles (recueillis chez une outarde, non identifiée, au Natal), nous donnons la description

(1) Travail effectué au Laboratoire de Zoologie (Vers) du Muséum National d'Histoire Naturelle de Paris (Bourse de l'Institut de Haute Culture du Portugal). matériel.

(2) Nous remercions vivement le Professeur J.-A. Travassos Santos Dias qui nous a cédé le 
de la femelle. La morphologie des spicu'es du mâle est précisęe, après dissection de ces organes.

\section{Matériel.}

Lot $\mathrm{n}^{\circ} 1230 / \mathrm{M}: 1 \delta^{\star}$ et 2 d'Hartertia natalensis, trouvés chez un spécimen de Lissotis melanogaster notophila, provenant de la région de Mahel, Circonscription de Magude, District de Gaza, Mozambique (4-4-1952).

Lot $\mathrm{n}^{\circ} 1231 / \mathrm{M}: 4 \uparrow$ adultes et 1 juvénile d'Hartertia natalensis, trouvés chez le même hôte, provenant de la même région (4-4-1952).

Lot $\mathrm{n}^{\circ} 1232 / \mathrm{M}: 2$ ô et 5 월 d'Hartertia natalensis et 1 ô d'H. zuluensis, trouvés aussi chez le même hôte et dans la même région (4-4-1952).

\section{Description.}

MÂle: Corps long de 31 à $35 \mathrm{~mm}$, large de 1,05 à $1,26 \mathrm{~mm}$.

Le spicule gauche, mince, est constitué dans la région distale par des ramifications complexes (fig. 1, G), toutes situées sur la face externe du spicule ; il existe schématiquement un tronc antérieur bifurqué, dirigé vers l'arrière et muni d'une grosse pointe récurrente ; les trois pointes du spicule dessinées par Mönnig correspondent à ces trois axes principaux ; les deux ensembles de ramifications sont reliés chacun par une membrane; la pointe du spicule est fine, parfois recourbée.

L'extrémité du spicule gauche d'H. natalensis diffère donc de celle d' $H$. zuluensis, redécrit par Kung (1948) ; chez cette espèce, les ramifications sclérifiées sont dispersées en éventail et soutenues par une seule membrane.

Le spicule droit est fortement sclérifié, avec une extrémité distale obtuse et bordée par une aile membraneuse.

Dimensions : chez un mâle long de $35 \mathrm{~mm}$, les dimensions sont les suivantes: deirides situées à $140 \mu$ de l'extrémité antérieure ; anneau nerveux et pore excréteur situés réspectivement à 590 et $540 \mu$; œsophage long de $5,9 \mathrm{~mm}$; ailes cervicales longues de $4,75 \mathrm{~mm}$; spicule gauche et spicule droit longs respectivement de 4,10 et $0,8 \mathrm{~mm}$; gubernaculum long de $160 \mu$ et ailes caudales de $1,67 \mathrm{~mm}$; queue longue de $0,85 \mathrm{~mm}$.

Femelle: La longueur des spécimens varie de 52 à $68 \mathrm{~mm}$, avec une largeur maximale de $1,55 \mathrm{~mm}$.

Le corps est incurvé ventralement dans la région antérieure; il est effilé aux extrémités, principalement dans la région caudale. Cuticule très épaisse et striée transversalement. La tête est isolée du reste du corps par un cou plus étroit, suivi d'un renflement cuticulaire net sur les faces dorsale et ventrale (fig. 1, A) ; elle est constituée par deux pseudolèvres, nettement trilobées; le rebord externe de chaque pseudolèvre est denticulé (fig. 1, B). Papilles disposées deux à deux; les papilles labiales externes sont légèrement plus grandes que les papilles céphaliques et entourées par un 

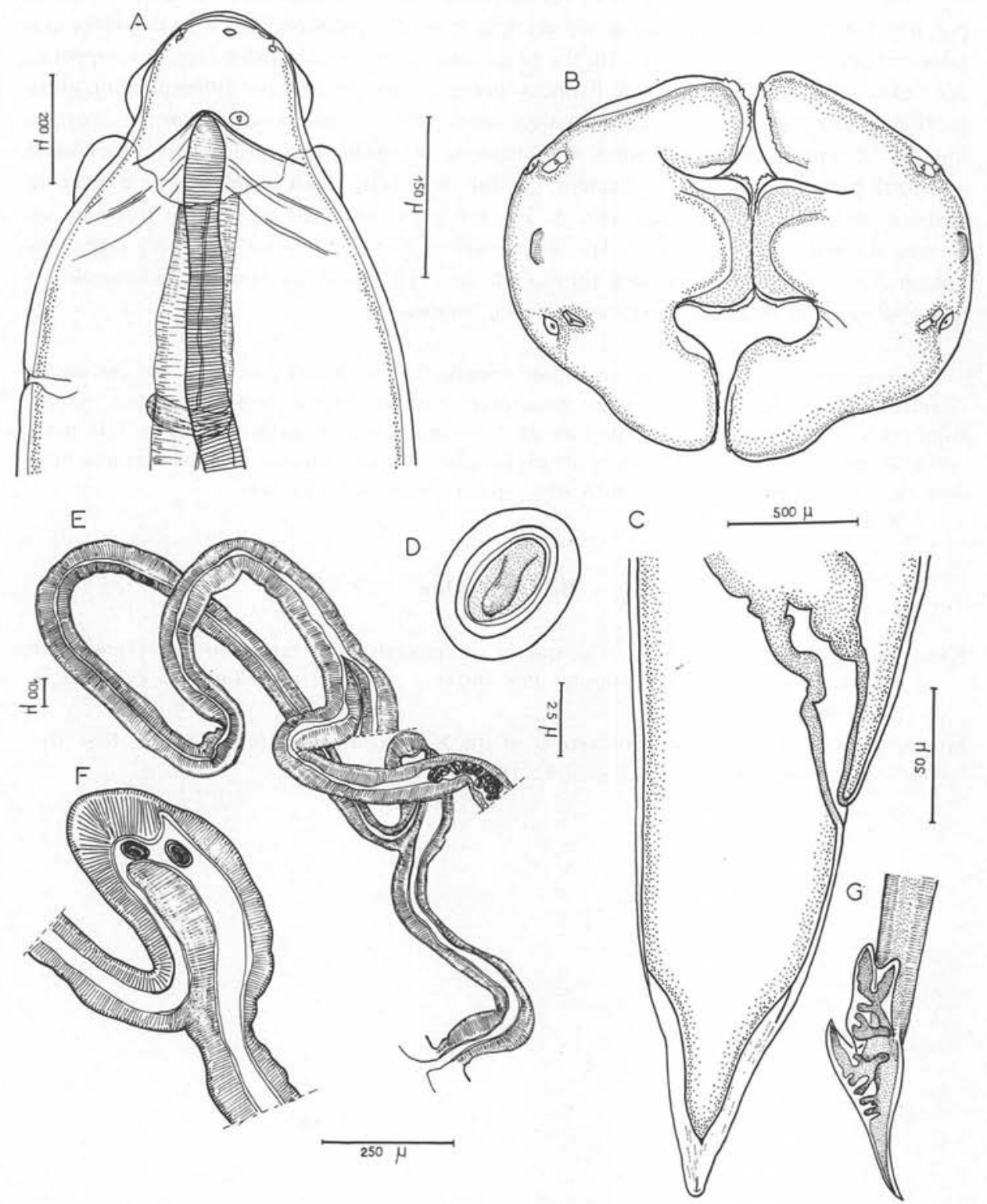

Fig. 1. - A à F : Femelle; $\mathrm{A}$ : région antérieure en vue latérale; $\mathrm{B}$ : tête, vue apicale; $\mathrm{C}$ : région caudale, vue latérale; D : œuf; E : ovéjecteur; F : sphincter; G : extrémité distale du spicule gauche. - A : éch. $200 \mu$; B : éch. $150 \mu$; C : éch. $500 \mu$; D : éch. $25 \mu$; E : éch. $100 \mu ; \mathrm{F}$ : éch. $250 \mu ; \mathrm{G}$ : éch. $50 \mu$ 
repli cuticulaire saillant (fig. 1, B); les amphides sont voiumineuses. Deirides formées par une forte pointe récurrente et situées à la base des pseudolèvres, à la naissance des ailes cervicales, mais déportées sur la face dorsale. Ailes cervicales très développées ; pore excréteur situé au niveau de l'anneau nerveux ; œsophage sans différenciation entre portion glandulaire et musculaire; vulve située près du milieu du corps; la portion impaire de l'ovéjecteur est longue et comprend le sphincter; celui-ci est simplement constitué par une anse de l'ovéjecteur qui fait une saillie bien visible ; à ce niveau, la lumière de l'ovéjecteur s'élargit (fig. $1, \mathrm{~F}$ ) ; les œufs mûrs ont une coque très épaisse, formée de deux couches (fig. 1, D) ; ils mesurent 55-62,5 $\times 40-47,5 \mu$; les embryons ont un crochet subterminal et une râpe céphalique. La queue est conique ; la cuticule est très épaisse dans la moitié postérieure de sa longueur.

Dimensions: les dimensions chez une femelle longue de $64 \mathrm{~mm}$ sont les suivantes: deirides situées à $200 \mu$ de l'extrémité antérieure ; anneau nerveux et pore excréteur respectivement à 775 et $710 \mu$; æsophage long de $7,70 \mathrm{~mm}$; ailes cervicales longues de $5,35 \mathrm{~mm}$; vulve située à $28,20 \mathrm{~mm}$ de l'extrémité céphalique ; portion impaire de l'ovéjecteur longue de $6,12 \mathrm{~mm}$ et sphincter long de $0,55 \mathrm{~mm}$; queue longue de $1,57 \mathrm{~mm}$.

\section{Bibliographie}

KUNG (C. C.), 1948. - On some new species of spirurids from terrestrial vertebrates, with notes on Habronema mansoni, Physaloptera paradoxa and Hartertia zuluensis. J. Helm., 22 (3-4), 141-164.

MöNNIG (H. O.), 1931. - Two new species of the Nematode genus Hariertia. 17th Rep. Dir. Vet. Serv. Dept. Agric. Union S. Africa, 1, 269-272. 